\title{
Numerical Study of Heat and Mass Transfer during the Evaporative Drying of Porous Media
}

\author{
Karima SELLAMI ${ }^{1 *}, M^{\prime}$ 'barek FEDDAOUI ${ }^{2}$, Nabila LABSI $^{1}$, M'hand OUBELLA $^{2}$, Youb Khaled BENKAHLA ${ }^{1}$ \\ ${ }^{1}$ Laboratoire des Phénomènes de Transfert, FGMGP, USTHB, Bab Ezzouar, Alger, Algérie. \\ ${ }^{2}$ Laboratoire Génie de l’Energie, Matériaux et Systèmes (LGEMS), ENSA Agadir, Maroc.
}

\begin{abstract}
The paper deals with numerical study of drying process of porous media of sand during the evaporation of a liquid saturated porous layer within parallel vertical channel. The liquid and air streams are modeled as two coupled laminar boundary layers incorporating non-Darcian models of the inertia and boundary effects. The governing equations and the associated boundary conditions are discretized by means of the finite volume method implemented on a staggered mesh and the velocity-pressure coupling is processed by the SIMPLER algorithm. The influences of the inlet mass flow of the drying gas, porous layer thickness and the porosity on the drying process are analyzed. Results show that the drying rate of the porous media is improved by the reduction of the porosity and porous layer thickness a large drying rate is obtained with high inlet mass flow and high inlet gas temperature.
\end{abstract}

\section{Nomenclature}

$C$ inertia parameter.

$C p \quad$ specific heat of the fluid at constant pressure, $\left(J \cdot \mathrm{kg}^{-1} \cdot \mathrm{K}^{-1}\right)$

$d \quad$ porous layer thickness, (m)

$D_{h} \quad$ hydraulic diameter, $(m)$

$D \quad$ mass diffusivity, $\left(m^{2} \cdot s^{-1}\right)$

$g \quad$ gravitational acceleration, $\left(m \cdot s^{-2}\right)$

$H \quad$ channel width, $(m)$

$h_{f g} \quad$ latent heat of vaporization, $\left({\left.\mathrm{J} . \mathrm{kg}^{-1}\right)}^{-1}\right.$

$K \quad$ permeability of the porous layer, $\left(\mathrm{m}^{2}\right)$

$k \quad$ thermal conductivity, $\left(W \cdot m^{-1} \cdot K^{-1}\right)$

$L \quad$ channel length, $(m)$.

$m_{L} \quad$ liquid mass flow rate, $\left(\mathrm{kg} \cdot \mathrm{m}^{-1} \cdot \mathrm{s}^{-1}\right)$

$m_{I} \quad$ evaporative mass flux, $\left(\mathrm{kg} \cdot \mathrm{m}^{-2} \cdot \mathrm{s}^{-1}\right)$

$P \quad$ pressure, $\mathrm{Pa}$

$Q_{l} \quad$ latent heat flux $\left(\mathrm{W} \cdot \mathrm{m}^{-2}\right)$

$Q_{s} \quad$ sensible heat flux $\left(\mathrm{W} \cdot \mathrm{m}^{-2}\right)$

Re Reynolds number

$T \quad$ temperature, $(K)$

$U_{0} \quad$ gas inlet velocity, $\left(m \cdot s^{l-}\right)$

$V_{x} \quad$ longitudinal velocity, $\left(m \cdot s^{-1}\right)$

$V_{y} \quad$ transverse velocity, $\left(m \cdot s^{-1}\right)$

$W \quad$ mass vapour fraction,

$x \quad$ longitudinal coordinate, $(m)$

$y \quad$ transverse coordinate, $(m)$

\section{Greek symbols}

$\beta \quad$ coefficient of thermal expansion, $\left(K^{-1}\right)$

$\varepsilon \quad$ porosity

$\phi \quad$ relative humidity of the air-vapour mixture.

$\mu \quad$ dynamic viscosity, $\left(\mathrm{kg} \cdot \mathrm{m}^{-1} \cdot \mathrm{s}^{-1}\right)$.

$\rho$ density, $\left(\mathrm{kg} \cdot \mathrm{m}^{-3}\right)$

$\Gamma \quad$ gas mass flow $\left(\mathrm{kg} \cdot \mathrm{m}^{-1} \cdot \mathrm{h}^{-1}\right)$

\begin{tabular}{ll}
\multicolumn{2}{l}{ Indices } \\
$a$ & and exponents \\
$e$ & air \\
$I$ & interface \\
$L$ & liquid \\
$m$ & average \\
$v$ & vapour \\
$S$ & solid \\
$w$ & wall \\
0 & inlet
\end{tabular}

\section{Introduction}

Drying process is one of the most energy-intensive and important processes in the industry and engineering. In order to improve the performance of these processes and the use of energy, it is therefore essential to develop efficient energy systems for the drying. The evaporative process is still nowadays an alternative and sustainable solution for the processing of porous materials. However, the study of evaporative drying is therefore fundamental to understand and ultimately develop solutions for these processes.

Ilic et al. [1] proposed a model to describe the drying process of a semi-infinite bed of green pellets by forced convection of hot dry air. Chen et al. [2] proposed a mathematical model for drying processes involves simultaneous heat, mass and momentum transfer. These models are based on equations of conservation of energy, conservation of mass and conservation of momentum together with the necessary physical and transport properties. The temperature and moisture distributions obtained by the mathematical model are compared favorably with the reported experimental results.

\footnotetext{
* Corresponding author: sellami_karima $@$ yahoo.fr
} 
A theoretical analysis of the effect of drying on the rising damp equilibrium is proposed by I'anson et al. [3], the authors concluded that with the presence of strong drying conditions the rising damp problem may be reduced and various practical steps may be taken to assist such drying. Atakan et al. [4] presented a mathematical model for heat and mass transfer analyses of the drying process and they compared the theoretical study to the experimental data. Hall et al. [5] confirmed the existence of two stages of drying by analysing the processes of evaporation and drying of porous building materials experimentally. The results show that the rate of drying is strongly influenced by both airflow and temperature.

A recent work has focused on the effect of capillaritydriven viscous flow through macroscopic liquid films during the isothermal drying of porous materials is proposed by Yiotis et al. [6]. A mathematical model is presented and the results are generalized to drying under an applied temperature gradient. Shokri et al. [7] presented an experimental study to investigate different mechanism occurring during the evaporative drying of sand. The authors conclude that decreasing drying rate was limited by vapor exchange between progressively drying surface and the viscous boundary layer above. Later the same authors [8] proposed a composite characteristic length for predicting drying front with a high and constant drying rates from layered porous media.

The main motivation of the present study is to examine numerically the range of parameters, which improve the drying process of porous material of sand. The influences of the inlet drying gas mass flow, porous layer thickness and the porosity on the heat and mass transfers are discussed. The purpose of this study, is fall within the scope of the search for a better understanding of drying process to improve its performance.

\section{Analysis and modelling}

As shown schematically in Figure 1, the problem concerns a vertical channel of two parallel plates, separated by distance $\mathrm{H}$, the left wall is covered by a thin porous layer of thickness $d$ and it is wetted by a falling liquid film with an inlet temperature $\mathrm{T}_{\mathrm{L} 0 \text {, and inlet flow }}$ rate $\mathrm{m}_{\mathrm{L} 0}$. The second plate is adiabatic and dry. A laminar descending flow of humid air flowed throng the channel with a uniform velocity $\mathrm{U}_{0}$, temperature $\mathrm{T}_{\mathrm{G} 0}$ and relative humidity $\phi_{0}$. The heat is transferred from the drying air stream to the porous plat.

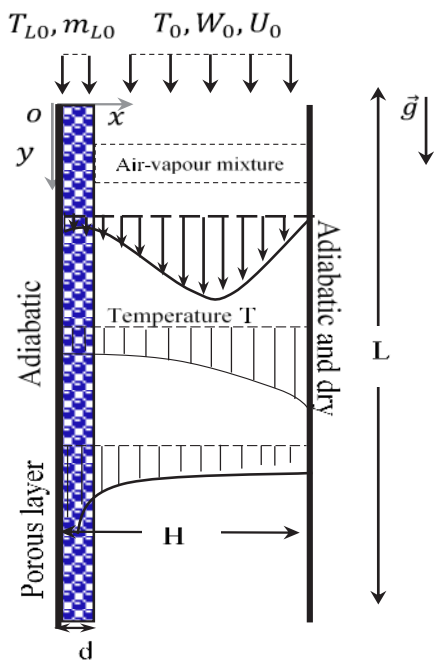

Fig.1: Schematic presentation of the physical problem.

\subsection{Governing equations}

\subsubsection{Basic equations for the liquid film}

Adopting non-Darcian models, the laminar axial momentum and energy boundary layer equations can be further simplified as follow:

$$
\begin{aligned}
& 0=-\rho_{L} g+\frac{\partial}{\partial y}\left(\frac{\mu_{L}}{\varepsilon} \frac{\partial V_{x}}{\partial y}\right)-\frac{\mu_{L}}{K} V_{x}-\frac{\rho_{L} C}{\sqrt{K}} V_{x}^{2} \\
& \frac{\partial}{\partial x}\left(\rho_{L} V_{x} C_{p, L} \mathrm{~T}\right)=\frac{\partial}{\partial y}\left(k_{e} \frac{\partial \mathrm{T}}{\partial y}\right)
\end{aligned}
$$

The effective thermal conductivity $\mathrm{k}_{\mathrm{e}}$ is defined as $k_{e}=\varepsilon k_{L}+(1-\varepsilon) k_{S}$.

\subsubsection{Basic equations for gas flow}

The two-dimensional laminar continuity, and conservation equations can be written as:

$$
\begin{aligned}
& \frac{\partial\left(\rho_{m} V_{x}\right)}{\partial x}+\frac{\partial\left(\rho_{m} V_{y}\right)}{\partial y}=0 \\
& \frac{\partial\left(\rho_{m} V_{x}^{2}\right)}{\partial x}+\frac{\partial\left(\rho_{m} V_{x} V_{y}\right)}{\partial y}=-\frac{\partial p}{\partial x}+\rho_{m} g+\frac{\partial}{\partial x}\left(\mu_{m} \frac{\partial V_{x}}{\partial x}\right)+\frac{\partial}{\partial y}\left(\mu_{m} \frac{\partial V_{x}}{\partial y}\right) \\
& \frac{\partial\left(\rho_{m} V_{y} V_{x}\right)}{\partial x}+\frac{\partial\left(\rho_{m} V_{y}^{2}\right)}{\partial y}=-\frac{\partial p}{\partial y}+\frac{\partial}{\partial x}\left(\mu_{m} \frac{\partial V_{y}}{\partial x}\right)+\frac{\partial}{\partial y}\left(\mu_{m} \frac{\partial V_{y}}{\partial y}\right) \\
& \frac{\partial\left(\rho_{m} V_{x} C_{p} \mathrm{~T}\right)}{\partial x}+\frac{\partial\left(\rho_{m} V_{y} C_{p} \mathrm{~T}\right)}{\partial y}=\frac{\partial}{\partial x}\left(k_{m} \frac{\partial \mathrm{T}}{\partial x}\right)+\frac{\partial}{\partial y}\left(k_{m} \frac{\partial \mathrm{T}}{\partial y}\right) \\
& \frac{\partial\left(\rho_{m} V_{x} W\right)}{\partial x}+\frac{\partial\left(\rho_{m} V_{y} W\right)}{\partial y}=\frac{\partial}{\partial x}\left(\rho_{m} D_{m} \frac{\partial W}{\partial x}\right)+\frac{\partial}{\partial y}\left(\rho_{m} D_{m} \frac{\partial W}{\partial y}\right)
\end{aligned}
$$

\subsection{Boundary conditions}

At the inlet: $\quad x=0$ 
For the gas

$$
V_{y, m}=0, \quad T=T_{0, m}, \phi=\phi_{0, m}
$$

For the liquid $0<y<d$

\section{At the walls:}

$$
T=T_{0, L} \quad m_{L}=m_{0, L}
$$

$$
\begin{array}{ccc}
y=0 & V_{x, L}=0 & \left.\frac{\partial T}{\partial y}\right|_{y=0}=0 \\
y=H & \mathrm{~V}_{x, m}=V_{y, m}=0, & \left.\frac{\partial T}{\partial y}\right|_{y=H}=\left.\frac{\partial W}{\partial y}\right|_{y=H} 0
\end{array}
$$

At liquid-air interface $(y=d)$ :

$$
\begin{aligned}
& V_{x, I}=V_{x, I, m}=V_{x, I, L}, \quad T_{\mathrm{I}}=T_{I, m}=T_{I, L} \\
& \tau_{I}=\left[\mu \frac{\partial V_{x}}{\partial y}\right]_{I, L}=\left[\mu \frac{\partial V_{x}}{\partial y}\right]_{I, m} \\
& {\left[-k_{e} \frac{\partial T}{\partial y}\right]_{I, L}=\left[-k \frac{\partial T}{\partial y}\right]_{I, m}+\dot{m}_{I} \cdot h_{f g}} \\
& V_{e}=-\left.\frac{D_{m}}{1-W_{I}} \frac{\partial W}{\partial y}\right|_{I}
\end{aligned}
$$

At the outlet: $d<y<H$ and $x=L$

$$
\frac{\partial V_{x}}{\partial x}=\frac{\partial V_{y}}{\partial x}=\frac{\partial T}{\partial x}=\frac{\partial W}{\partial x}=0
$$

The inlet mass flow rate $m_{0, L}$ of the liquid film is given by Bird et al. [9]

$$
m_{0, L}=\frac{\rho_{l} g}{3 v_{L}} d^{3} \varepsilon^{3}
$$

Bulk gas temperature and mass fraction

$$
\begin{aligned}
& T_{\text {bulk }}=\int_{d}^{H} \rho_{m} T \cdot V_{x} \cdot d y / \int_{d}^{H} \rho_{m} \cdot V_{x} \cdot d y \\
& W_{\text {bulk }}=\int_{d}^{H} \rho_{m} W \cdot V_{x} \cdot d y / \int_{d}^{H} \rho_{m} \cdot V_{x} \cdot d y
\end{aligned}
$$

Relative humidity

$$
\varphi=\frac{P_{v}}{P_{v}^{s a t}(T)}
$$

The evaporative mass flow is defined by:

$$
m_{I}(x)=\rho_{m} V_{e}=\left.\frac{-\rho_{m} D_{m v}}{1-W_{I}} \frac{\partial W}{\partial y}\right|_{I}
$$

The sensible and latent heat fluxes are given by:

$$
\begin{aligned}
& Q_{S}=-\left.k_{m} \frac{\partial T}{\partial y}\right|_{I} \\
& Q_{l}=-\left.\rho_{m} \frac{D_{m v} h_{f g}}{1-W_{I}} \frac{\partial W}{\partial y}\right|_{I}
\end{aligned}
$$

\section{Numerical method}

For this study, the governing equations (1)-(7) coupled with the boundary conditions mentioned above were solved using a finite volume method proposed by Patankar [10]. The velocity-pressure coupling is treated with the SIMPLER algorithm.

The computational code has been validated by comparing our results with those of Chou et al. [11] in the case of heat and mass transfer enhancement of liquid film evaporation obtained by introducing a liquidsaturated porous layer in an inclined channel under the following conditions:

$$
\begin{aligned}
& T_{0}=T_{L 0}=27^{\circ} \mathrm{C}, \mathrm{p}_{0}=1 \mathrm{~atm}, T_{W}=60^{\circ} \mathrm{C} \text { and } \\
& H=0.05 \mathrm{~m} ; d=0.01 \mathrm{~m}, \phi_{0}=70 \% .
\end{aligned}
$$

Figure 2 shows good agreement between our results and those of Chou et al. [11], as the relative difference is less than $3 \%$.

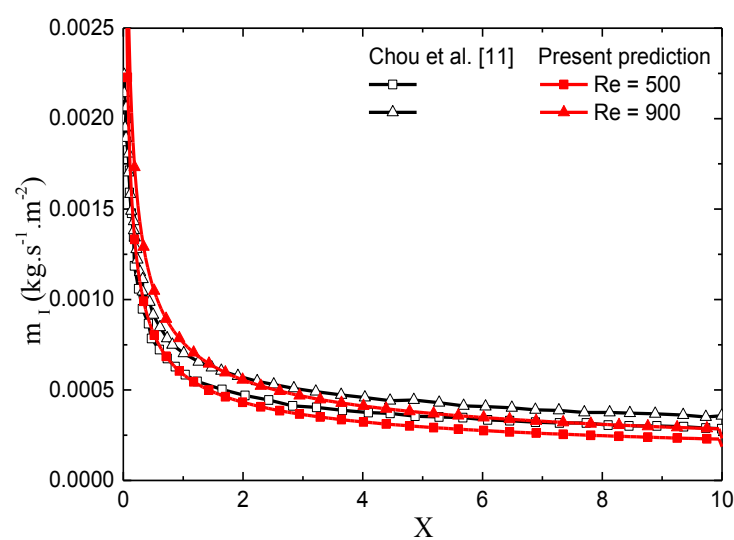

Fig. 2: Axial distributions of mass evaporation rate ( $\varepsilon=0.4$,

$$
d=0.01 \mathrm{~m}) \text {. }
$$

\section{Results and discussion}

The study is performed to analyses the drying mechanism of the porous layer of sand by studying the effect of the inlet gas mass flow, porous layer thickness $d$ and the porosity $\varepsilon$ on the evaporative drying process of porous material in vertical channel of length $L=3 \mathrm{~m}$ and width $H=0.02 \mathrm{~m}$. All the results of this study have been obtained for the case of inlet relative humidity $\phi_{0}=$ $10 \%, T_{G 0}=60^{\circ} \mathrm{C}, T_{L l}=20^{\circ} \mathrm{C}$.

In order to determine the optimal parameters for an efficient drying of a sand porous medium, we study in this section the effect of the porous medium characteristic (porosity $\varepsilon$ and porous layer thickness $d$ ) and the inlet drying gas mass flow $\Gamma_{G 0}$ on the evaporative drying. For this, the evolution of the mass evaporation and drying rate with those parameters is illustrated in Figures 3 and 4 respectively.

As showing in Figure 3, the mass evaporation is improved by the decrease of the porosity and the porous layer thickness, that the decrease of the porosity ameliorates the effective conductivity and therefore improve the heat and mass exchange between two media and favorite the drying of the porous medium. Additionally, the increase of the porous layer thickness increases the imbibing liquid flow and therefore decreases the evaporation rate. 


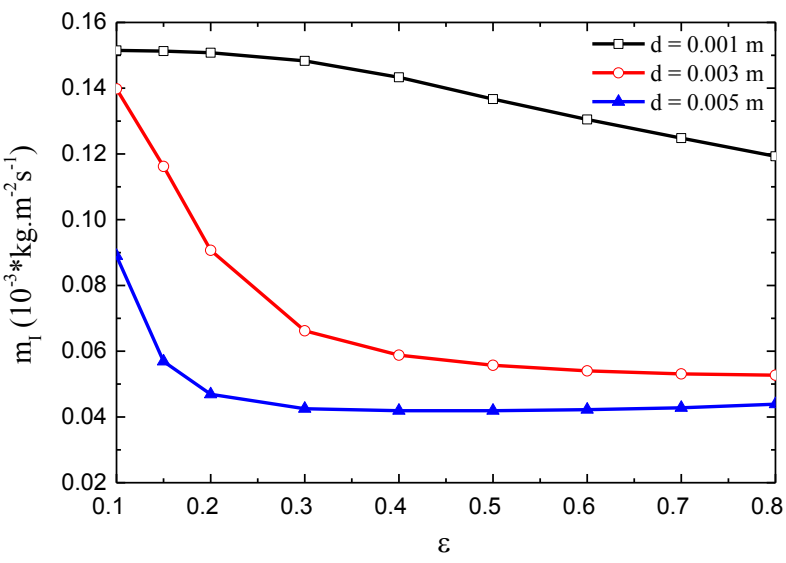

Fig. 3: Evolution of the mass evaporation as a function of porosity for various porous layer thicknesses. $\left(T_{G 0}=60^{\circ} \mathrm{C}, T_{L l}\right.$ $\left.=20^{\circ} \mathrm{C}, \quad \phi_{0}=10 \%, \quad \Gamma_{G 0}=100 \mathrm{~kg} \cdot \mathrm{m}^{-1} \cdot \mathrm{h}^{-1}\right)$

Figure 4 shows the effect of the inlet drying gas mass flow and the inlet gas temperature on the drying rate of the porous layer. As depicted in this figure it is obvious that the evaporative drying is improved with the increase of the gas inlet mass flow and the increase of the inlet gas temperature. The increase of the gas mass flow increases the gas particles at the porous layer-gas interface, which increases the heat and mass exchange between the two media. This implies that higher inlet gas flow allows the absorption of greater quantity of water vapour, consequently the enhancement of the evaporative drying along the channel.

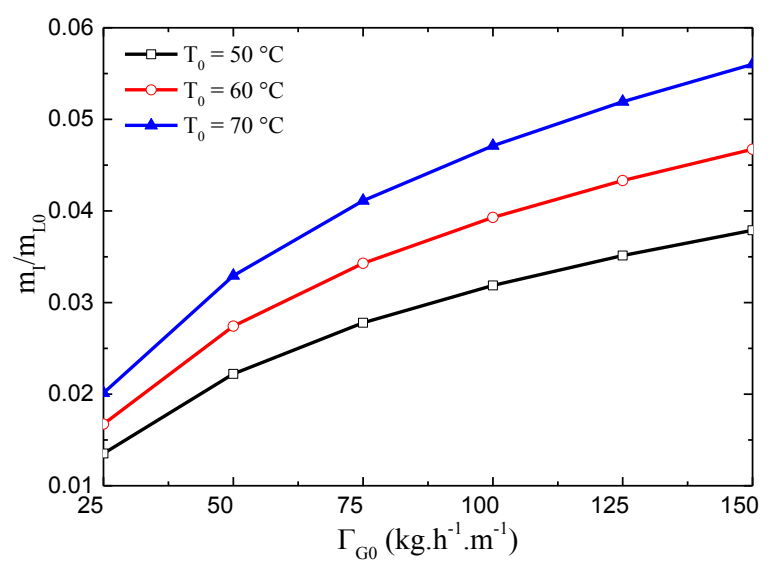

Figure 4

Evolution of the drying rate as a function of inlet gas mass flow for various inlet gas temperature. $\left(T_{L l}=20^{\circ} \mathrm{C}\right.$,

$$
\phi_{0}=10 \%, \varepsilon=0.1, \mathrm{~d}=0.001 \mathrm{~m} \text { ) }
$$

To more understand the mechanism of the evaporative drying of the porous materials, we present the evolution of the heat exchange flux between the two media at the porous layer-gas interface as function of the parameter selected above.

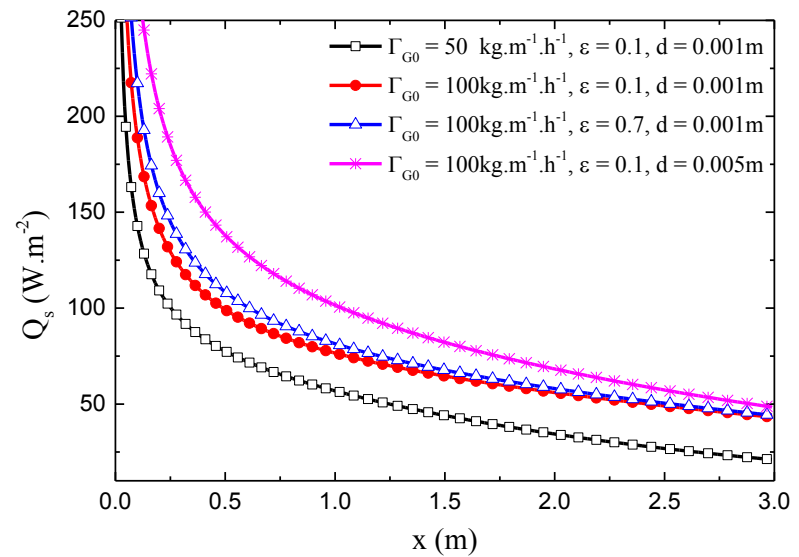

Fig. 5: Evolution of the sensible heat flux along the channel. $\left(T_{G 0}=60^{\circ} \mathrm{C}, T_{L l}=20^{\circ} \mathrm{C}, \phi_{0}=10 \%\right)$

The heat exchange representing by the sensible and the latent heat flux is shown in Figures 5 and 6, respectively. The curves show an intense heat exchange near the liquid gas interface. Moreover, in this figure we note that the heat exchange is strongly influenced by the porosity and the inlet drying gas flow. Hence a larger sensible and latent heat fluxes are obtained for higher inlet gas flow and higher porosity.

We can also see, on those figures, that the latent Nusselt number is more important than the sensible one, which means the predominance of latent mode transfers for the select conditions.

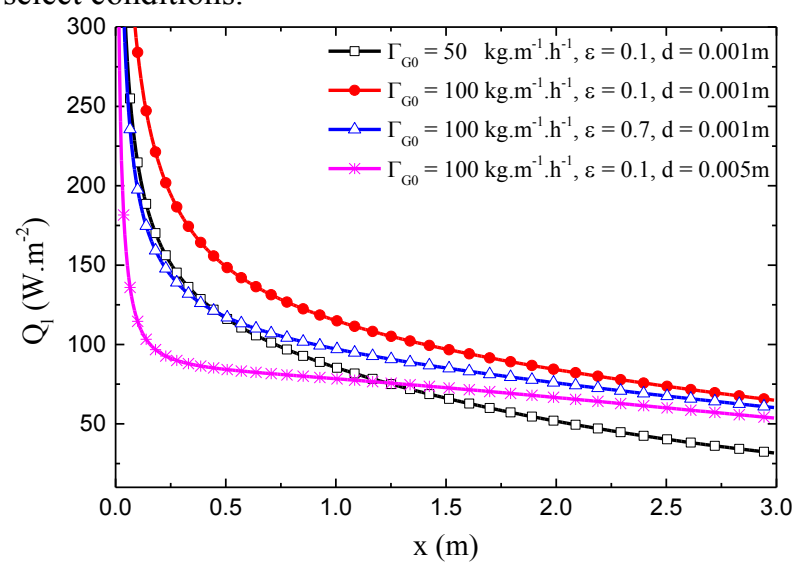

Fig. 6: Evolution of the latent heat flux along the channel. $\left(T_{G 0}\right.$ $=60^{\circ} \mathrm{C}, T_{L l}=20^{\circ} \mathrm{C}, \phi_{0}=10 \%$ )

The evolution of the gas average temperature and the mass fraction in the gas stream along the channel for various inlet drying gas flow $\left(\Gamma_{G 0}=50 \mathrm{~kg} \cdot \mathrm{m}^{-1} \cdot \mathrm{h}^{-1}, 100\right.$ $\left.\mathrm{kg} . \mathrm{m}^{-1} \cdot \mathrm{h}^{-1}\right)$, porous layer thickness $(d=0.001 \mathrm{~m}, 0.005 \mathrm{~m})$ and porosity $(\varepsilon=0.1,0.7)$ is shown in figure 7 . For all the studied cases we note a monotonic decrease of the gas average temperature indicate that the gas flow exchanges by convection the heat necessary for drying the porous media, this decrease is more intense for the case of high inlet drying gas flow $\left(\Gamma_{G 0}=100 \mathrm{~kg} \cdot \mathrm{m}^{-1} \cdot \mathrm{h}^{-1}\right)$ and lower porosity $(\varepsilon=0.1)$. This figure shows also that the decrease of porous layer thickness $d$ improve heat transfer. 
As we can see also in this figure the decrease of the average gas temperature is accompanied by an increase in the average mass fraction, implicate the evaporation of the liquid film at the liquid-gas interface. We note, also, that the evaporation is enhanced owing to lower porosity $\varepsilon$. The later ameliorates the effective conductivity whenever it is low and therefore improves the heat and mass transfer between the two media.

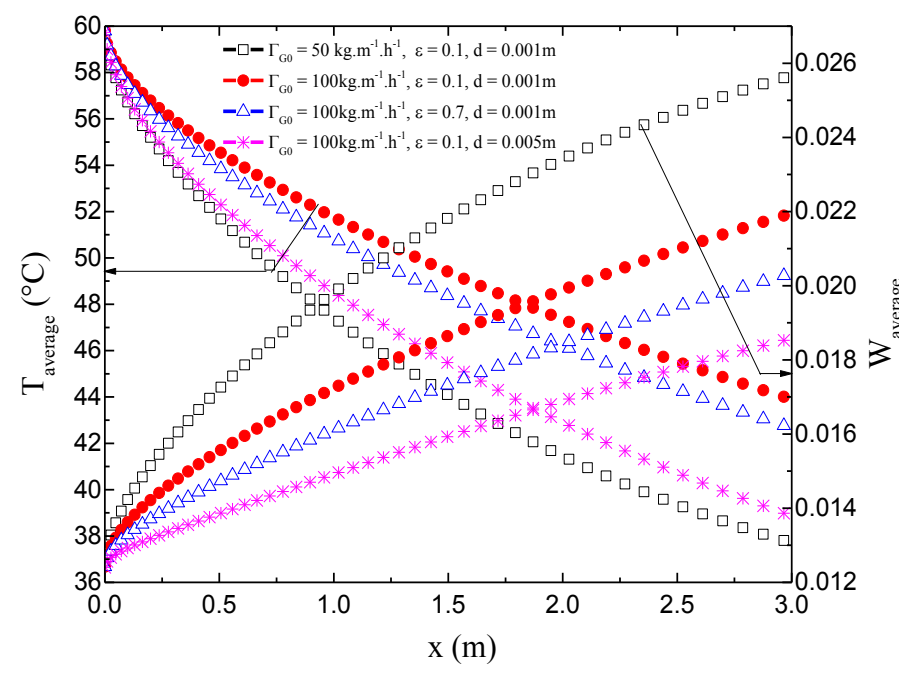

Fig. 7: Evolution of average gas temperature and mass fraction along the channel. $\left(T_{G 0}=60^{\circ} \mathrm{C}, T_{L l}=20^{\circ} \mathrm{C}, \phi_{0}=10 \%\right)$

Figure 8 presents the axial evolution of the relative humidity for various inlet drying gas flow $\left(\Gamma_{G 0}=50\right.$ $\left.\mathrm{kg} \cdot \mathrm{m}^{-1} \cdot \mathrm{h}^{-1}, 100 \mathrm{~kg} \cdot \mathrm{m}^{-1} \cdot \mathrm{h}^{-1}\right)$, porous layer thickness $(d=$ $0.001 \mathrm{~m}, 0.005 \mathrm{~m})$ and porosity $(\varepsilon=0.1,0.7)$, the relative humidity increase along the channel. In addition to the heat exchange, the gas serves to remove moisture from the wet porous medium in the gas diffusion through the evaporation mechanism inducing an increase in moisture of the gas stream.

This figure indicates, also, that the relative humidity decreases with increasing the inlet air velocity and the porosity.

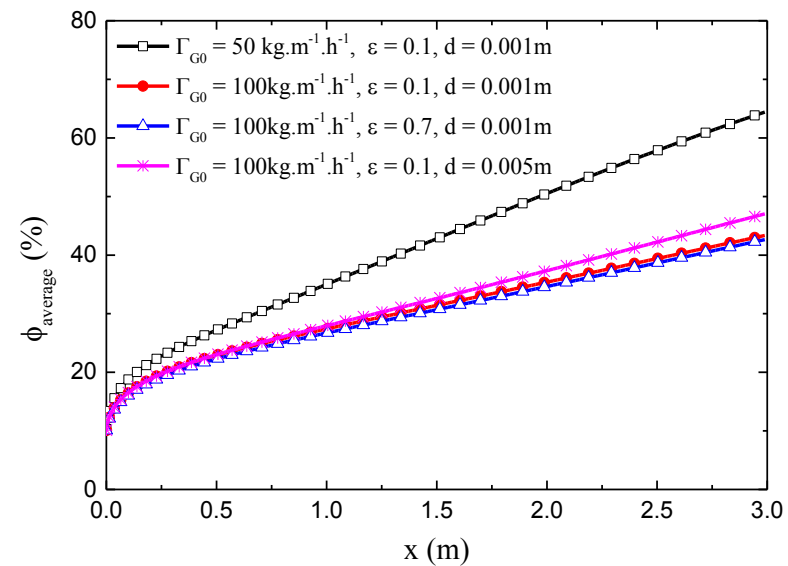

Fig. 8: Evolution of the relative humidity along the channel. $\left(T_{G 0}=60^{\circ} \mathrm{C}, T_{L l}=20^{\circ} \mathrm{C}, \phi_{0}=10 \%\right)$

\section{Conclusion}

This paper presents a two dimensional numerical study to describe heat and mass transfer during the evaporative drying of porous media. The wet Sand layer was considered as a saturated porous medium. The effect of the imposed inlet drying gas mass flow, porous layer thickness and porosity on the drying performance of porous materials has been analysed. The main conclusions are summarized as follows:

- The evaporative drying is enhanced by the increase of the inlet air flow.

- Lower porosity or less thicker porous layer foster the evaporation, and therefore the drying of the porous materiel.

The parametric study carried out made it possible to highlight the influence of the inlet drying gas mass flow and the characteristics of porous media on the drying performance. The results obtained from this study permitted to conclude that the drying rate is improved following the increase of the air flow and the reduction of the porosity. The high mass flow of the drying gas cause a larger sensible and latent heat transfer as well as a better heat and mass exchange occurs and large drying rate is noted.

\section{KEYWORDS}

Drying, evaporation, wet porous layer, finite volume methods.

\section{References}

1. M. Ilic and I.W. Turner, Drying of a material, Appl. Math. Modelling, 10, pp. 16-24 (1986).

2. P. Chen, C. David, T. PEI, A mathematical model of drying processes, Int. J. Heat and mass Transfer 32, pp. 297-310 (1989).

3. S. J. I'anson, W.D. Hoff, Water Movement in Porous Building Materials-VIII. Effects of Evaporative Drying on Height of Capillary Rise Equilibrium in Walls, Building and Environment, 21, pp. 195-200 (1986).

4. A. Avci, M. Can, A. B. Etemoglu, A theoretical approach to the drying process of thin film layers, Applied Thermal Engineering, 21, pp. 465-479, (2001).

5. C. Hall, W. D. Hoff, M. R. Nixon, Water Movement in Porous Building Materials-VI. Evaporation and Drying in Brick and Block Materials, Building and Environment, 19, pp. 13-20, (1984).

6. A. G. Yiotis, A. G. Boudouvis. A. K. Stubos, I. N. Tsimpanogiannis. and Y. C. Yortsos, Effect of Liquid Films on the Drying of Porous Media, American Institute of Chemical Engineers, 50, pp. 2721-2737 (2004). 
7. N. Shokri, P. Lehmann, P. Vontobel and D. Or, Drying front and water content dynamics during evaporation from sand delineated by neutron radiography, Water Resources Research, 44, doi: 10.1029/2007WR006385 (2008).

8. N. Shokri, P. Lehmann, and D. Or, Evaporation from layered porous media, Journal of Geophysical Research, 115, doi: 10.1029 /2009JB006743 (2010).

9. R. Bird, W. Stewart, and E. Lightfoot, Transport phenomena, 2 ed. JohnWiley, New York (2002).

10. S. V. Patankar, Numerical heat transfer and fluid flow, Hemisphere (1980).

11. Y. Chou, R.J. Yang, the evaporation of a saturated porous layer inside an inclined airflow channel, Int. J. Heat Fluid Flow, 28, pp.407-417 (2007). 